\title{
Rapidly Evolving Structures in the Photosphere of the Mira Variable TX Cam.
}

\author{
P.J.Diamond
}

MERLIN/VLBI National Facility, Jodrell Bank Observatory, University of Manchester, Macclesfield, Cheshire, SK11 9DL, U.K.

\section{A.J.Kemball}

National Radio Astronomy Observatory, PO Box 0, Socorro, NM 87801, $U S A$

\begin{abstract}
VLBA observations of the $43 \mathrm{GHz}$ SiO masers in the circumstellar envelope surrounding the Mira variable TX Cam reveal dramatic structural variations over the 80 week stellar cycle. The dominant dynamical mode is one of expansion although other complex motions are visible. The gravitational field of the star does not have a significant effect on the dynamics observed, these are probably governed more by the magnetic field and the effects of the shock waves resulting from the pulsation of the Mira itself.
\end{abstract}

\section{Introduction}

Maser emission from $\mathrm{SiO}$ occurs in the envelopes that surround Mira variables that result from a period of significant mass-loss. The excitation temperature of the $43 \mathrm{GHz} \nu=1, J=1-0 \mathrm{SiO}$ maser transition places it close to the central star (Elitzur 1992). This is also the region in which the mass-loss process is most prominent, with material being lost from the nearby stellar surface into a region permeated by shocks, local temperature and density gradients, magnetic fields and other phenomena.

This paper reports on a continuing VLBA monitoring study of the $\mathrm{v}=1$, $\mathrm{J}=1-0 \mathrm{SiO}$ masers towards TX Cam. TX Cam lies at a distance of $317 \mathrm{pc}$ (Patel, Joseph \& Ganesman, 1992), its spectral classification lies between M8 and M10 over a pulsation period of 557 days. The star exhibits strong $\mathrm{SiO}$ maser emission, in several vibrational and rotational states (e.g. Jewell et al., 1987).

\section{Observations}

We observed the $v=1, J=1-0 \mathrm{SiO}$ maser emission towards TX Cam approximately bi-weekly from 24 May $1997(\phi=0.72)$ until 19 Feb $1999(\phi=$ 1.83) using the Very Long Baseline Array (VLBA) operating at $43 \mathrm{GHz}$. The details of the observations and automated data processing system are given in Diamond \& Kemball (2001). 
The principal result generated by the project to date is a time sequence of images, a movie, showing the global evolution of the structure of the maser emission in the circumstellar envelope. Each frame of the movie was generated from the Stokes I image cube of an epoch by running through the cube in velocity space and extracting the pixel with maximum intensity at each value of Right Ascension and Declination. The composite image formed represents the total structure well. The movie comprises a total of 44 epochs, an animated GIF file showing the movie can be found at:

http://www.jb.man.ac.uk/ pdiamond/txcam44.gif
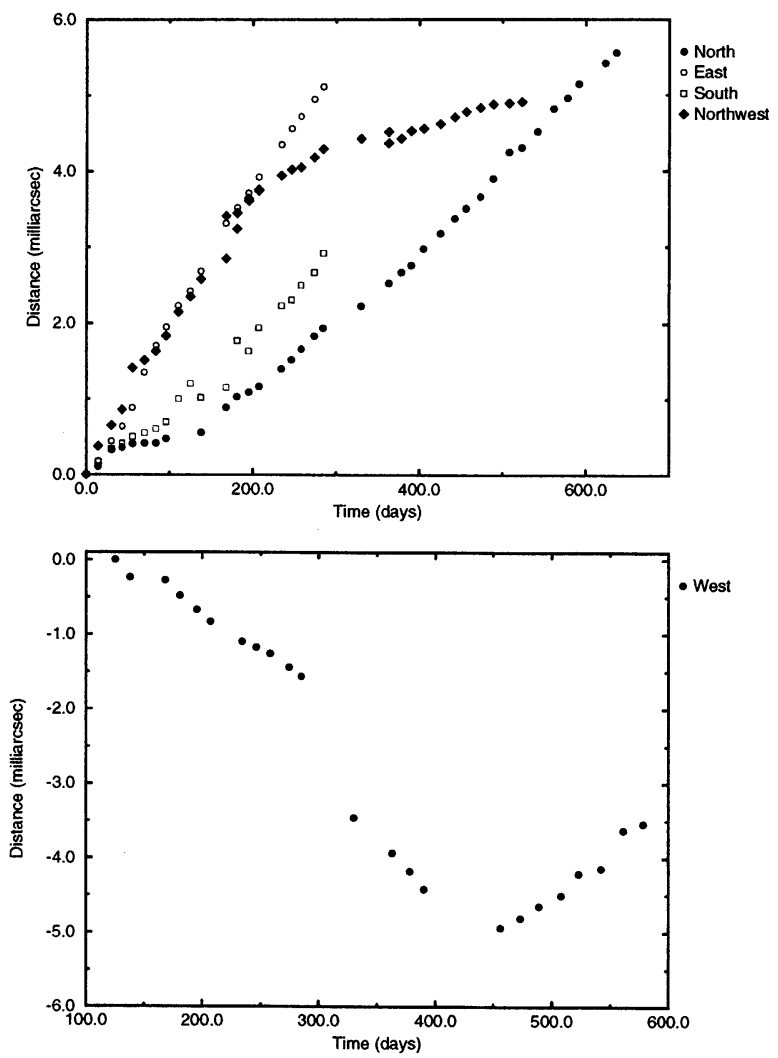

Figure 1. Diagrams showing the proper motions of components or groups of components in various regions of the $\mathrm{SiO}$ ring. The upper frame shows the motion experienced by the majority of maser features. The lower shows that some features fall back towards the star and then 'bounce'.

\section{Proper Motions}

Study of the movie shows that, as our series of observations proceeded, the shape of the $\mathrm{SiO}$ structure changed considerably as the position of the maser 
emission moved outwards. Expansion is quite clearly the dominant dynamical mode, although areas in the west and southwest of the ring contain inward moving masers and other complex motions. By the final epoch of this series of observations we observe a tightly defined ring of masers, visible in all quadrants bar the northwest, and approximately $20 \%$ larger than the ring seen in the first epoch.

In addition to the global changes of the envelope structure we are able to monitor the evolution of individual features or groups of features. We chose compact, single features in each quadrant of the image; an additional feature in the clearly decelerating structure in the northwest of the envelope was also selected. Due to the short lifetime of individual $\mathrm{SiO}$ maser features it was not always possible to follow a single maser for a significant time. In that case, as one maser died an adjacent maser was used to follow the motion of a particular cloud. Such feature tracking was necessary in the western and northwestern complexes.

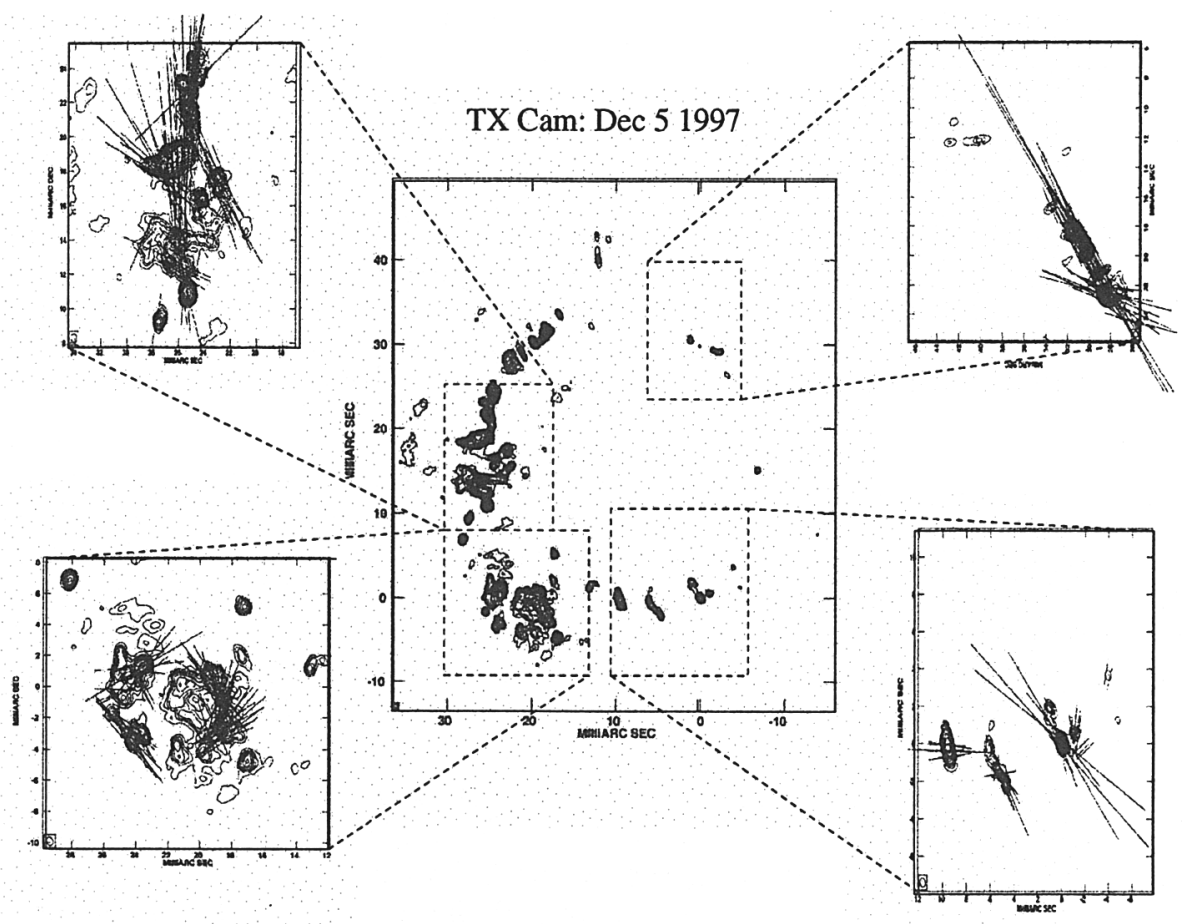

Figure 2. A montage of images of TX Cam. The central image shows the total intensity structure as seen on Dec 51997 . The four surrounding images show the linear polarization structure of the regions marked. These regions are discussed further in the text.

Figure 1a shows the results of the monitoring of four of the five masers we followed. As can be clearly seen the masers tend to follow well-defined paths but with a variety of velocities. The feature on the western side of the envelope 
moves outwards at a constant velocity of $10.1 \mathrm{~km} / \mathrm{s}$ for $\sim 300$ days and then fades away. In contrast, the group of features in the NE complex move with an initial velocity of $9.5 \mathrm{~km} / \mathrm{s}$ and decelerate quadratically to $1.8 \mathrm{~km} / \mathrm{s}$. Figure $1 \mathrm{~b}$ shows the path followed by a feature in the structure that lies on the eastern side of the ring. It can clearly be seen to fall inwards towards the star at a velocity of $\sim 10 \mathrm{~km} / \mathrm{s}$ and then, at optical phase $\sim 1.3$ it 'bounces' and begins to move outwards at $\sim 7.5 \mathrm{~km} / \mathrm{s}$.

The complex motions are indicative of several effects. The appearance of a new ring and the reversal of direction of the component on the eastern side of the ring are strong indicators of the effect of a new shock wave reaching the region containing $\mathrm{SiO}$ masers. If the shock wave was emitted at optical maximum this implies it has a velocity of $\sim 15 \mathrm{~km} / \mathrm{s}$ which may be at odds with single-dish $\mathrm{SiO}$ data which suggest a somewhat higher value.

The deceleration of the material in the NE quadrant implies that the gas containing the $\mathrm{SiO}$ emission is ploughing into unseen material presumably deposited in the previous stellar cycle. The fact that we see such deceleration in this one region alone tells us that the stellar dynamics are not controlled by the gravitational field of the star.

\section{Polarization}

Kemball and Diamond (1997) made the first polarization image of $\mathrm{SiO}$ and demonstrated that the linear polarization vectors lay predominantly tangential to the maser ring. Through a measurement of the circular polarization in the ring they determined a value for the magnetic field at the location of the $\mathrm{SiO}$ of $B \sec \Theta \sim 5-10 \mathrm{G}$.

In this contribution we show the polarization structure as revealed by observations on Dec 5, 1997 (see Figure 2). It is clear from the main image and the closeups of structures in the NE, NW and SW quadrants that the tangential structure is still dominant. This is possibly the result of the compression of the gas and its embedded magnetic field caused by a passage of a shock wave.

We note a very peculiar structure revealed in the polarization image of the confused emission seen in the SE quadrant. In total intensity the region appears to have no discernable structure and it varies and moves with no recognizable pattern in the movie. However, the polarization structure in Fig. 2 reveals a ring-like pattern. The linear polarization vectors form a tangential pattern similar to, but considerably smaller then, the pattern seen throughout the ring. The origin of this structure is unknown.

Acknowledgments. NRAO is operated by Associated Universities, Inc., under cooperative agreement with the National Science Foundation.

\section{References}

Jewell, P.R., Dickinson, D.F., Snyder, L.E. \& Clemens, D.P. 1987, ApJ323, 749

Kemball.A.J. \& Diamond, P.J. 1997 ApJ481, L111

Patel, N.A., Joseph, A., \& Ganesman R. 1992 J. Astrophys. Astr., 13, 241 